\title{
Terahertz Detection by the Entire Channel of High Electron Mobility Transistors
}

\author{
M. Sakowicz ${ }^{a}$, J. Łusakowski ${ }^{a}, \mathrm{~K} . \mathrm{Karpierz}^{a}, \mathrm{~W} . \mathrm{KnAP}^{a, *}$, \\ M. GrynberG ${ }^{a}$, K. Köhler ${ }^{b}$, G. VAlusis $^{c}$, K. Golaszewska ${ }^{d}$, \\ E. Kamińska ${ }^{d}, \mathrm{~A} . \mathrm{PiotrowskA}^{d}, \mathrm{P} \cdot \mathrm{CABAN}^{e}$ \\ AND W. StRUPińsKI ${ }^{e}$ \\ ${ }^{a}$ Institute of Experimental Physics, University of Warsaw \\ Hoża 69, 00-681 Warsaw, Poland \\ ${ }^{b}$ Fraunhofer-Institut für Angewandte Festkörperphysik \\ D-79108 Freiburg, Germany \\ ${ }^{c}$ Semiconductor Physics Institute, A. Goštauto 11, Vilnius LT-01108, Lithuania \\ and Institute of Materials Science and Applied Research, Vilnius University \\ Sauletekio av. 9, LT-10222, Vilnius, Lithuania \\ ${ }^{d}$ Institute of Electron Technology, al. Lotników 32/46, 02-668 Warsaw, Poland \\ ${ }^{e}$ Institute of Electronic Materials Technology \\ Wólczyńska 133, 01-919 Warsaw, Poland
}

GaAs/AlGaAs and GaN/AlGaN high electron mobility transistors were used as detectors of $\mathrm{THz}$ electromagnetic radiation at liquid helium temperatures. Application of high magnetic fields led to the Shubnikov-de Haas oscillations of the detection signal. Measurements carried out with a simultaneous modulation of the intensity of the incident $\mathrm{THz}$ beam and the transistor gate voltage showed that the detection signal is determined by the electron plasma both in the gated and ungated parts of the transistor channel. This result is of importance for understanding the physical mechanism of the detection in high electron mobility transistors and for development of a proper theoretical description of this process.

PACS numbers: 75.75.+a, 78.20.Ls, 85.30.Tv, 07.57.Kp

*also at: Tohoku University RIEC Ultra-broadband Signal Processing, 2-1-1 Katahira, Aoba-ku 980-8577, Japan and GES-CNRS URM 5650, Université Montpellier 2, Place Eugène Bataillon, 34950 Montpellier, France 


\section{Introduction}

Field effect transistors (FETs) are known to be detectors of THz electromagnetic radiation both at liquid helium and room temperatures [1-5]. Illumination of the transistor with $\mathrm{THz}$ radiation leads to the generation of a constant drainsource voltage. This photovoltage is the detection signal, $S$, which is usually measured as a function of the gate voltage $U_{\mathrm{GS}}$, drain current, or temperature.

The idea of using transistors as detectors of $\mathrm{THz}$ radiation was put forward by Dyakonov and Shur [1] who showed that oscillations of the electron plasma in the transistor channel can couple to the external radiation. The physical mechanism of the detection is the rectification of plasma oscillations induced by the incident radiation in the transistor channel. The rectification process is possible because of the asymmetry of the channel (drain-gate vs. source-gate capacitances) and a nonlinear character of plasma wave excitations.

The model of the plasma instability [1] and the detection [2] was developed for a transistor with the gate covering the whole distance between the source and the drain. It is the only model which has been used so far to interpret experimental data, in spite of the fact that experiments were carried out on transistors with the gate covering the whole source-drain distance (i.e., Si metal-oxide-semiconductor FETs) or on high electron mobility transistors (HEMTs) with the gate covering only a small part of the channel. On the other hand, in the case of HEMTs, one can expect a strong coupling of the plasma in the gated and ungated parts of the channel. If it is so, the detection signal in HEMTs should be influenced by plasma excitations in the ungated part of the channel, too.

The aim of the present experiment was to clarify the role of the gated and ungated parts of the transistor channel in the detection of electromagnetic radiation with HEMTs. To this end, we used HEMTs processed on GaAs/AlGaAs and GaN/AlGaN heterostructures. Measurements were carried out at liquid helium temperatures and high magnetic fields with a simultaneous modulation of the incident $\mathrm{THz}$ light intensity and the gate voltage. This "double modulation" technique and application of quantizing magnetic fields allowed us to show unambiguously that the detection signal is influenced both by the gated and ungated plasma. This result is important for understanding of the physical mechanism of detection by the electron plasma in HEMTs. It also indicates that the model of detection traditionally used should be further developed to take into account the coupling of the plasma in the gated and ungated parts of the transistor channel.

\section{Experimental and results}

Experiments were carried out at $4.4 \mathrm{~K}$ on HEMTs processed on a high quality GaAs/AlGaAs and GaN/AlGaN heterostructures. In the case of GaAs FET, the gate length and the source-drain distance was equal to 0.8 and $10 \mu \mathrm{m}$, respectively. In the case of GaN FET, these numbers were 0.7 and $3 \mu \mathrm{m}$. Transistors were placed in a superconducting coil and they were cooled with a helium exchange gas; the 
magnetic field $(B)$ was perpendicular to the channel plane and was changed up to $8 \mathrm{~T}$. THz excitation was generated with a molecular $\left(\mathrm{CH}_{3} \mathrm{OH}\right)$ laser pumped with a $\mathrm{CO}_{2}$ laser. We used three laser lines: 1.84, 2.52 and $3.11 \mathrm{THz}$. The THz radiation was conducted to the sample by inox tubes and was neither intentionally focalized nor polarized.

The laser beam was modulated with a mechanical chopper (frequency $f_{L}=$ $8 \mathrm{~Hz}$ ) giving a 100\% (on-off) modulation of the beam intensity with the duty cycle 1:1. The gate potential was simultaneously modulated with a frequency $f_{G}=$ $1 \mathrm{kHz}$ and the gate polarization was $U_{\mathrm{GS}}+u \cos \left(2 \pi f_{G} t\right)$, with $u=10 \mathrm{mV}$. The aim of application of this double modulation technique was to measure the derivative of the detection signal with respect to the gate voltage, i.e., the derivative of the difference of the source-drain voltage measured with the $\mathrm{THz}$ light on and off. To measure that derivative we used two lock-in amplifiers. A lock-in connected between the source and the drain (L1) was referenced with $f_{G}$ and its integration time constant was $10 \mathrm{~ms}$. Since the laser beam was on and off during subsequent intervals of $1 / 16 \mathrm{~s}$, the L1 measured a derivative of the source-drain voltage (with respect to the gate polarization) in each of these intervals. The output of L1 changed every $1 / 16 \mathrm{~s}$ and was directed to the lock-in L2, referenced with $f_{L}$, which thus measured the derivative $\mathrm{d} S / \mathrm{d} U_{\mathrm{GS}}$.

To check the consistency of the method applied, we also used the third lock-in, L3, which was connected between the source and the drain and referenced with the frequency $f_{L}$; thus, L3 measured the detection signal $S$ with a standard lock-in technique. We verified that a numerical derivative of the signal $S$ measured with L3 as a function of $U_{\mathrm{GS}}$ was always proportional to the signal of L2 vs. $U_{\mathrm{GS}}$.

Examples of typical experimental data are shown in Fig. 1 and Fig. 2.

Figure 1 shows results for the GaAs/AlGaAs transistor. The thin line is the transfer characteristics which allows us to determine the threshold voltage $U_{\mathrm{TH}}$ to be about $-1 \mathrm{~V}$ and indicates a correct functioning of the transistor. The detection signal $S$ (measured with lock-in L3) at $2.52 \mathrm{THz}$ is plotted with a thick line. It shows a strong increase with the gate voltage approaching $U_{\mathrm{TH}}$, which is a typical behavior of the detection signal observed in experiments. The inset to Fig. 1 shows how the measured signal depends on the magnetic field. Oscillations which develop at $B$ higher than about $1.5 \mathrm{~T}$ are periodic in $B^{-1}$ which indicates that they are the Shubnikov-de Haas oscillations (SdHO). An interesting feature of the oscillations is that their apparent period does not depend on the gate voltage, i.e., on the electron concentration in the gated part of the channel. Similar results were obtained for other radiation frequencies used.

The data obtained for the GaN/AlGaN transistor is qualitatively the same as that presented in Fig. 1 with the exception that SdHO develop at larger magnetic field of about $5 \mathrm{~T}$ (due to the electron mobility smaller in GaN than in GaAs). Figure 2 shows an example of "double modulation" data, i.e., the derivative of the detection signal, as a function of the magnetic field. One can clearly observe a 


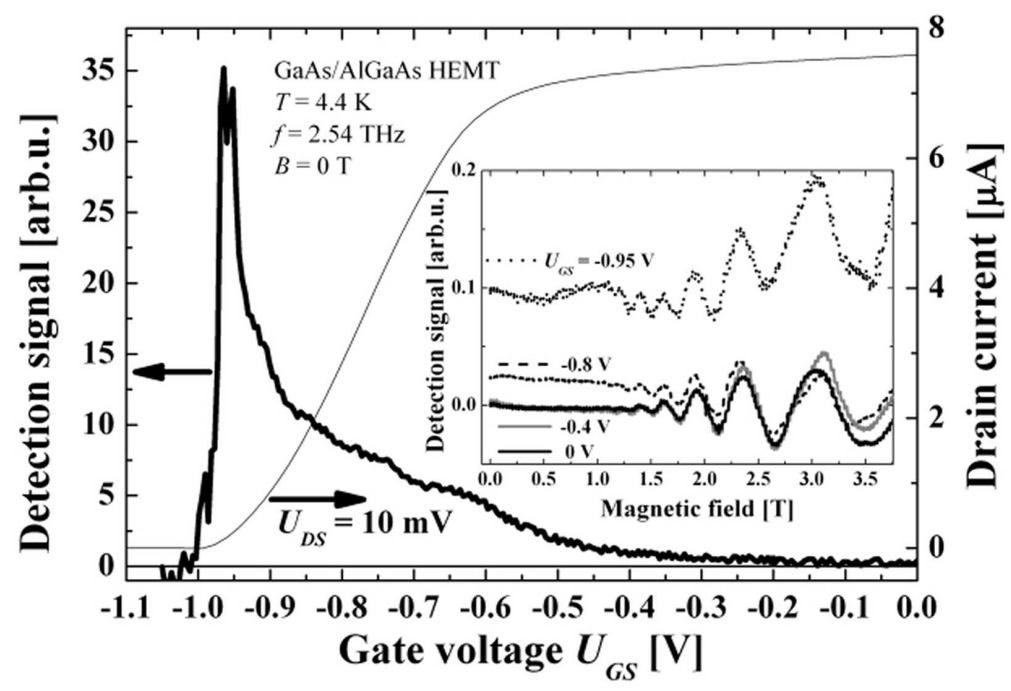

Fig. 1. Right axis: transfer characteristics measured at the drain-source voltage of $10 \mathrm{mV}$; left scale: detection signal $S$ as a function of the gate voltage at $2.52 \mathrm{THz}$ and $B=0$ T. Inset: oscillations of the detection signal in the magnetic field at $U_{\mathrm{GS}}=0 \mathrm{~V}$, $-0.4 \mathrm{~V},-0.8 \mathrm{~V}$ and $-0.95 \mathrm{~V}$ (bottom to top).

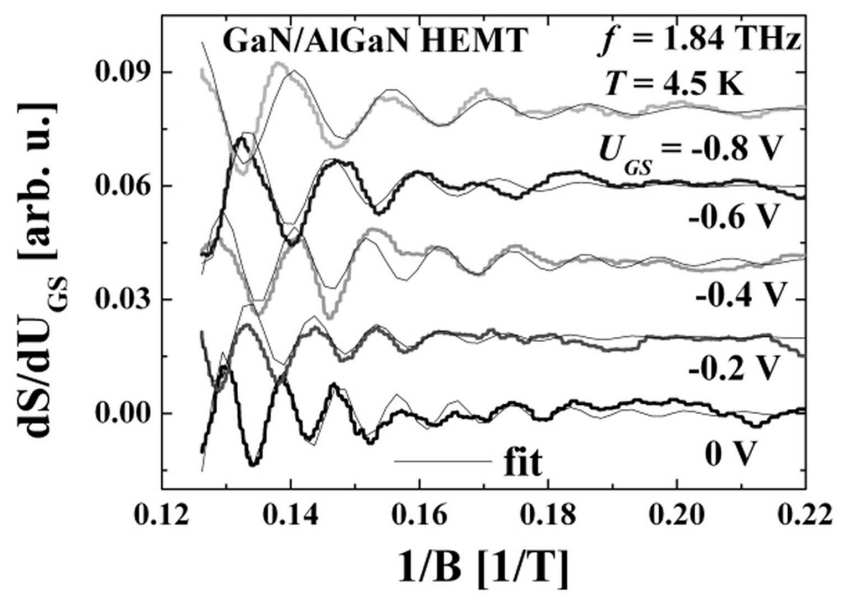

Fig. 2. Thick lines: oscillations of the derivative of detection signal at $1.84 \mathrm{THz}$ as a function of the inverse magnetic field at $U_{\mathrm{GS}}=0 \mathrm{~V},-0.2 \mathrm{~V},-0.4 \mathrm{~V},-0.6 \mathrm{~V},-0.8 \mathrm{~V}$ (bottom to top). Thin lines: numerical fit made simultaneously for all the curves with constraint of a linear dependence of the oscillation frequency on $U_{\mathrm{GS}}$.

dependence of the period of SdHO on the gate voltage $U_{\mathrm{GS}}$. Such a dependence was also observed in the case of GaAs/AlGaAs transistor when the derivative of the detection signal was plotted as a function of the inverse magnetic field. 


\section{Discussion}

A Fourier analysis of signals plotted in Fig. 2 shows that $\mathrm{d} S / \mathrm{d} U_{\mathrm{GS}}$ oscillates with a single frequency which linearly decreases when the gate voltage becomes more and more negative. On the other hand, a Fourier analysis of oscillations of the signal $S$ (Fig. 1) gives two frequencies: one of them does not depend on $U_{\mathrm{GS}}$ while the other coincides with the frequency of oscillations of $\mathrm{d} S / \mathrm{d} U_{\mathrm{GS}}$. These two oscillation frequencies correspond to electrons in the ungated and gated parts of the channel, respectively. In the case of transistors investigated, the contribution from the gated part is small and cannot be revealed just by inspection of the raw experimental data of $\mathrm{SdHO}$ of the signal $S$. Sometimes (it was not possible for GaN/AlGaN HEMTs) a gate-voltage dependent contribution to SdHO of the signal $S$ can be resolved with a Fourier analysis. On the other hand, SdHO of the derivative $\mathrm{d} S / \mathrm{d} U_{\text {GS }}$ contain only a voltage-dependent part. Thus, the double modulation technique allows us to amplify the contribution of the gated part of the channel and allows for its direct observation.

The same situation (two periods of SdHO - one not dependent on $U_{\mathrm{GS}}$ and the other linearly dependent) is observed in the case of magnetoresistance (MR) measurements with a concurrent gate voltage modulation [6]. We verified that $\mathrm{SdHO}$ in $S$ and $\mathrm{d} S / \mathrm{d} U_{\mathrm{GS}}$ show the same frequency as in standard MR measurements and MR with gate potential modulation, respectively. This proves that $\mathrm{SdHO}$ in the detection signal come from a modulation of the two-dimensional electron gas (2DEG) density with the magnetic field. Contrary to MR, SdHO in the detection signal in the magnetic field are not associated with any dc current flow through the channel of the transistor. In experiments, we do not intentionally bias the channel with any dc current nor voltage. Gate leakage current is below the detection level, much less than nA. Signal measured does not change when we use measurement setup with $10^{8} \Omega$ or $>10^{14} \Omega$ input impedance. When we apply source-drain current, we do not observe any signal growth. SdHO in $S$ and in its derivative are proposed to be related to the magnetic field induced oscillations of the parameter $\tau$ (scattering time) of the model of Ref. [2]. It would produce similar shape of $\mathrm{SdHO}$ as in MR measurements.

The presence of $\mathrm{SdHO}$ in detection signal associated both with gated and ungated part of 2DEG in the channel of the transistor proves that (i) 2DEG is crucial for the detection process; (ii) the 2DEG in the entire channel participates in the detection. This conclusion is true for both kinds of transistors investigated. However, up to now, no theoretical model has been developed which would predict how the detection signal depends on the gate voltage in the case of coupled regions of gated and ungated plasma. Our results indicate that further theoretical developments are necessary to describe correctly the process of detection of electromagnetic radiation in HEMTs.

In conclusion, high magnetic field studies of detection of $\mathrm{THz}$ radiation with GaAs and GaN HEMTs were carried out at liquid helium temperatures. A simul- 
taneous modulation of the laser light intensity and the gate voltage allowed us to show that the whole channel of the transistor (i.e., the gated and ungated parts) contributed to the detection signal. This result indicates that a new theoretical model should be proposed to analyze detection in HEMTs.

\section{Acknowledgments}

This work was supported by Polish 3T11B04528 and 162/THz/2006/02 and European Union MTKD-CT-2005-029671 grants. It was also supported by the CNRS and GDR-E project Semiconductor sources and detectors of THz frequencies and by the joint French-Lithuanian research programme Gilibert/EGIDE.

\section{References}

[1] M. Dyakonov, M.S. Shur, Phys. Rev. Lett. 71, 2465 (1993).

[2] M. Dyakonov, M.S. Shur, IEEE Trans. Electron Dev. 43, 380 (1996).

[3] W. Knap, F. Teppe, Y. Meziani, N. Dyakonova, J. Łusakowski, F. Boeuf, T. Skotnicki, D. Maude, S. Rumyantsev, M.S. Shur, Appl. Phys. Lett. 85, 675 (2004).

[4] R. Tauk, F. Teppe, S. Boubanga, D. Coquillat, W. Knap, Y.M. Meziani, C. Gallon, F. Boeuf, T. Skotnicki, C. Fenouillet-Beranger, D.K. Maude, S. Rumyantsev, M.S. Shur, Appl. Phys. Lett. 89, 253511 (2006).

[5] X.G. Peralta, S.J. Allen, M.C. Wanke, N.E. Harff, J.A. Simmons, M.P. Lilly, J.L. Reno, P.J. Burke, J.P. Eisenstein, Appl. Phys. Lett. 81, 1627 (2002).

[6] M. Sakowicz, R. Tauk, J. Lusakowski, A. Tiberj, K. Karpierz, Z. Bougrioua, M. Azize, P. Lorenzini, W. Knap, M. Grynberg, J. Appl. Phys. 100, 113726 (2006) 